\title{
First report of OXA-48 carbapenemase-producing Escherichia coli in Taiwan
}

\author{
Y Jao ${ }^{1 *}$, P-S Lee ${ }^{2}$, C-T Hung ${ }^{1}$, C-F Wang ${ }^{2}$, MH Hsieh ${ }^{1,3}$, C-Y Lin ${ }^{1,3}$, Y-H Chen ${ }^{1,3}$, P-L Lu ${ }^{2,3}$ \\ From 3rd International Conference on Prevention and Infection Control (ICPIC 2015) \\ Geneva, Switzerland. 16-19 June 2015
}

\section{Introduction}

Carbapenems are the last-line antibiotic in treating Enterobacteriaceae because its broad-spectrum antibacterial ability, high bactericidal activity and good stability to $\beta$-lactamases. However, carbapenem-resistant Enterobacteriaceae (CRE) infection increased all over the world in recent years. The challenge of carbapenems in treating Enterobacteriaceae is increasing.

\section{Objectives}

A 55-year old woman who had been diagnosed with cancer and had received multiple courses of chemotherapy in Vietnam, visited our hospital for a second opinion in June 2014.

Left breast tumor with non-discharge necrotic tissue was presented at admission. The laboratory examination demonstrated WBC 6300/uL, Hb $12.1 \mathrm{~g} / \mathrm{dL}$ and PLT 103000/uL. Specimen from left breast wound grew Escherichia coli, which resistant to carbapenems, broadspectrum $\beta$-lactams and fluoroquinolones. There was no inflammation reaction caused by the pathogen. During hospitalization, she did not receive antimicrobial therapy effective to the pathogen.

\section{Methods}

We found that this Escherichia coli had the presentation of carbapenemase via modified Hodge test. Polymerase chain reaction and DNA sequencing was performed to test if Carbapenemase (blaKPC, blaNDM, blaIPM, blaVIM, blaOXA) or $\beta$-lactamases (SH, TEM, OXA, GES, CTX-M) presents.

\section{Results}

Resistance genes of blaOXA-48 and CTX-M-1-group were found. Besides, this Escherichia coli also presented with loss of outer membrane protein A and F. With multilocus sequence typing (MLST) analysis, this Escherichia coli is ST-405.

\section{Conclusion}

To our knowledge, this is the first OXA-48 carbapenemase producing Escherichia coli isolated in Taiwan, though it's originated from Vietnam. It's resistance profile is similar to other OXA-48 carbapenemase producing Escherichia coli isolated from Japan and France. According to this finding, Escherichia coli acquiring OXA-48 carbapenemase may had spread to Southeastern Asia. In order to prevent the transmission of CRE, the detection of carbapenemase in Enterobacteriaceae is important.

\section{Disclosure of interest}

None declared.

\section{Authors' details \\ 'Department of Infection Control, Kaohsiung Medical University Hospital, Kaohsiung Medical University, Kaohsiung, Taiwan, Province of China. ${ }^{2}$ Clinical Microbiology Laboratory, Kaohsiung Medical University Hospital, Kaohsiung Medical University, Kaohsiung, Taiwan, Province of China. ${ }^{3}$ Division of Infectious Diseases, Department of Internal Medicine, Kaohsiung Medical University Hospital,Kaohsiung Medical University, Kaohsiung, Taiwan, Province of China.}

Published: 16 June 2015

doi:10.1186/2047-2994-4-S1-P126

Cite this article as: Jao et al:: First report of OXA-48 carbapenemaseproducing Escherichia coli in Taiwan. Antimicrobial Resistance and Infection Control 2015 4(Suppl 1):P126. 